\title{
A Comparative Study on Decentralized Storage Platforms for Self-sovereign Data
}

\author{
Yoon-Jin Han1), Geun-Hyung Kim²)
}

\begin{abstract}
With the rising interest in social media and file- and video-sharing applications, the demand for storage platforms has increased significantly. In these applications, our data are stored on centralized storage platforms owned and operated by giant service providers. These platforms, however, have been facing problems such as data breaches, data outrages, rising storage costs, lack of ownership, censorship, and monitoring. A new concept of a decentralized storage platform has emerged to solve these issues. Several platforms have been devised based on this concept. In this study, we aim to present a survey of the leading decentralized storage platforms currently implemented on the Internet. To this end, we first derived the requirements and four essential elements of a decentralized storage platform. We then investigated the major leading decentralized storage platforms in terms of blockchain usage, data decentralization method, data encryption method, and data integrity verification. As a result, we found that a decentralized storage platform requires an extra function of security and protection for data and has the potential to provide self-sovereign data.
\end{abstract}

Keywords: Decentralized Storage, Decentralized Data, Blockchain, Peer-to-peer, Encryption

\section{Introduction}

Recently, with the rising interest in social media and file- and video-sharing applications, the demand for storage platforms has increased significantly. In these applications, our data are stored on centralized storage platforms owned and operated by giant service providers. This kind of solution, however, comes with problems such as data breaches, data outrages, rising storage costs, lack of ownership, censorship, and monitoring[1].

If a failure occurs on the centralized server, the service on the platform may be paralyzed or data may be lost. Amazon Web Services(AWS), for example, experienced the disruption of 190 of its services stopped at the same time due to an $11-\mathrm{h}$ error. In the event of a service error,

Received(November 11, 2019), Review Result(1st: December 13, 2019, 2nd: January 28, 2020), Accepted(April 25, 2020)

1) (Student) 47340 Dept. Digitalmedia Engineering, Dong-Eui Univ., Busanjin-gu, Busan, Korea email: qkr030@naver.com

2) (Professor, Corresponding Author) 47340 Dept. Game Engineering Major, Dong-Eui Univ., Busanjin-gu, Busan, Korea

email: geunkim@deu.ac.kr 
users cannot find the cause but have to merely rely on the centralized storage platforms that provide the services[2].

Bottlenecks can appear when the traffic spikes as the server can only have a finite number of open ports for connections from client nodes. When high traffic occurs, the centralized server can essentially suffer a denial-of-service attack or distributed denial-of-service attack[3].

Personal information and data on the centralized storage platform may be provided to a third party without the owner's permission. Dropbox's data is encrypted through Advanced Encryption Standard(AES) but it is not safe from data censorship because the decryption key for the encrypted data is held by the servers[4].

Decentralized storage platforms of different kinds have recently emerged to solve these problems. A decentralized storage platform encrypts and distributes data across a decentralized network. Users are in control of private encryption keys and of their own data. No signups, or centralized servers are involved. No third party can access or control user's data, unlike in traditional centralized storage platforms. The objective of the present study is to survey the main decentralized storage platforms currently implemented and deployed on the Internet.

Decentralized storage platforms should meet the following criteria[5-8]. The decentralized storage platform should ensure that no single server controls the entire service. Users should receive data that are distributed and saved on request. In addition, centralized servers should not manage data. Users should retain ownership of their data and be the only ones to be able to access their data or give permission to other users to do so. The network should operate smoothly so that users can access the data at any time. The decentralized storage platform should provide a reliable storage method for users when data is distributed and saved. Finally, it should be less expensive than centralized storage platforms.

In the present study, we derived the requirements and basic elements for the decentralized storage platforms. We then performed a comparative investigation of the major decentralized storage platforms in terms of blockchain usage, data decentralization method, data encryption method, and data integrity verification.

\section{Basic Elements of the Decentralized Storage Platforms}

The structure of a decentralized storage platform in four layers is depicted in [Fig. 1] Application of the 'Using the Storage' layer allows users to use a decentralized storage platform. Second, in the 'Users have ownership of data' layer, decentralized network, but not save data in a single server, and only users access data. The 'Data security' layer uses 
blockchain and data encryption to store data in a secure and transparent way. Finally, the 'Increasing trust' layer provides a reliable storage method for users when data is distributed and saved.

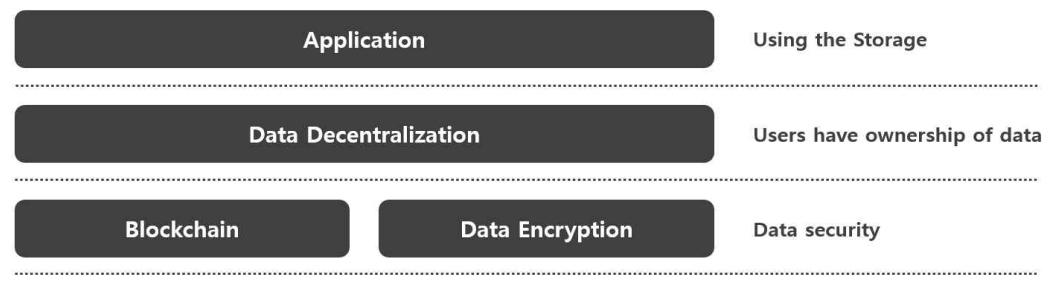

Data Integrity Verification

Increasing trust

[Fig. 1] Structure of a Decentralized Storage Platform

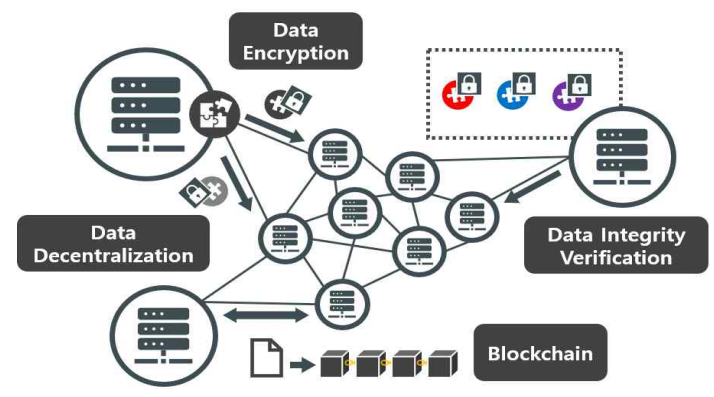

[Fig. 2] Decentralized Storage Platform

Before comparing various decentralized storage platforms, this section describes the four essential elements of decentralized storage platforms, as shown in [Fig. 2].

\subsection{Blockchain}

Blockchains are immutable digital ledger systems implemented in a distributed fashion and usually without a central authority. Some of the decentralized storage platforms are based on blockchain technology. Storage providers earn in cryptocurrency by providing data storage. Conversely, users pay storage providers to store data. In addition, blockchain technology can enhance security. It detects problems during data transmission through blockchain and responds to data errors and incorrect access to data[9].

Using the blockchain, users form smart contracts with storage providers. These contracts set pricing, uptime commitments, and other aspects of the relationship between the users and the 
storage providers. Since smart contracts are automatically enforced by the network, there is no need for trusted third parties[10].

\subsection{Data Decentralization}

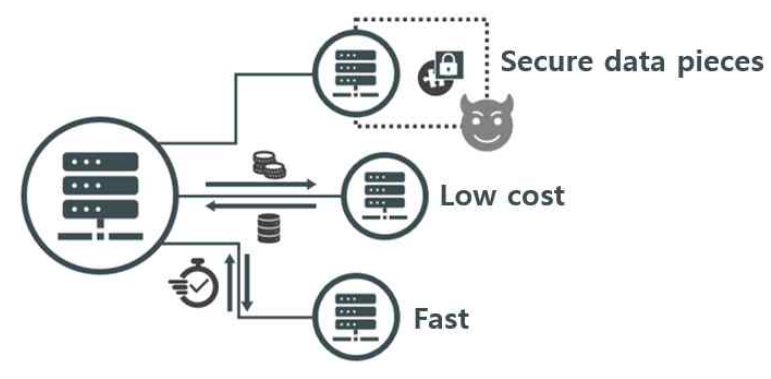

[Fig. 3] Features of Data Decentralization

Decentralized storage platforms should save data by distributing several nodes participating in the decentralized network, rather than save data in a single server. Decentralized storage platforms require no central control; therefore, users can save data without relying on large-scale storage providers. From [Fig. 3], it can be seen that data chunks can be stored in nearby nodes and used quickly. Decentralization reduces the risk of data failures and interruptions and improves the level of storage security and privacy[11].

Centralized storage platforms require a large amounts of data servers that are regularly maintained; however, decentralized storage platforms require only a large number of users to join the network. Decentralized storage platforms can reduce the amount of data server establishments and operations; therefore, they can provide services at a low cost as shown in [Fig. 3][12].

\subsection{Encryption}

Each data chunk is encrypted. This technology differs from traditional cloud storage providers, such as Amazon, which do not encrypt user data by default. Decentralized storage platforms are more secure because storage providers only store encrypted data chunks, rather than whole data. It enhances security with client-side encryption, so that only the data owner can access the data itself. Encryption and hashing are performed automatically before uploading data and all data on the network are encrypted and anonymized. [Fig. 3] shows that even if the encrypted data is snatched, it cannot be verified because it is encrypted, and data is safe from 
hacking because it is divided and saved in the network[10].

\subsection{Data Integrity Verification}

Since the users save their data with an untrusted storage provider, they want evidence that the storage provider keeps the data safe during the promised storage period[13]. Most decentralized storage platforms test storage regularly to check for data loss. Merkle trees, which are one of the data integrity verification methods, make it possible to prove that a small data chunk is part of a larger dataset. The advantage of these proofs is that they are very small, no matter how large the original data is.

In the case of blockchain-based decentralized storage platforms, this is important because the proofs are stored permanently on the blockchain. They save the result of the data integrity verification in the blockchain, which is used to calculate the reputation of the storage provider. If the proof fails, it is disadvantageous to storage providers; therefore, storage providers should control data safety.

\section{Various Decentralized Storage Platforms}

This section describes various decentralized storage platforms that are focused on the basic elements of the typical decentralized storage platform described in Section 2.

\subsection{InterPlanetary File System(IPFS) / Filecoin}

IPFS is a protocol developed by Protocol Labs for the distributed web that creates and connects a network used by individuals without the centralized servers. Data chunks divided by IPFS have their unique hash values. Certain data can have a unique hash value because it uses an IPFS list that can delete overlapped data, which saves the hash value of the chunk and data size information[14].

IPFS saves data in the Merkle DAG structure. This is a variant of the Merkle tree data structure, which creates trees made by data hash values, and detects changes in data. When saving data, Merkle directed acyclic graph(DAG) is formed by embedding the addresses of other data in the storage. When data changes, the hash value of the data changes and all data in IPFS are immutable because the hash value of the tree root node makes a big change[15]. IPFS does not use blockchain technology. Like other decentralized storage platforms, IPFS 
decentralizes and saves data with several nodes participating in the network, but does not have an incentive system that makes nodes provide storage.

Filecoin was developed by applying an incentive system to the IPFS. Filecoin represents a cost unit used for protocols or payments. Users pay by Filecoin when renting data storage and the storage providers save the data and activate the network by compensating Filecoin when the data maintenance is successful[16].

When a contract is concluded between the user and the storage provider for data storage, the storage provider pays Filecoin as the contract collateral. If the proof fails when verifying data integrity, which is conducted regularly, the collateral is lost. The data integrity verification is based on the blockchain consensus protocol.

IPFS and Filecoin undergo AES encryption before data partitioning for data security. The encrypted data is divided and stored in the decentralized network after each hashing. If you pay more for storing data in Filecoin, you can adjust the number of data replications. This increases data redundancy and reduces the risk of data loss.

\section{$3.2 \mathrm{Sia}$}

Sia is a decentralized storage platform based on blockchain technology. The Sia platform divides data into 30 chunks before uploading, each targeted for distribution to hosts across the world. Data chunks are created using a technology called Reed-Solomon erasure coding. Erasure coding allows Sia to divide data in a redundant manner, where any 10 of 30 chunks can fully recover a user's data[17]. Before leaving a user's computer, each data chunk is encrypted. This ensures that the storage provider only stores encrypted user data chunks. Sia uses the Threefish algorithm, an open-source, secure, high-performance encryption standard.

Sia adopts a blockchain smart contract to conclude a file contract. This is the agreement between the user and storage provider, including information such as cost, duration of use, and penalties for breach of contract. At the time of the contracture agreement, the storage provider pays a collateral based on Siacoin, a cryptocurrency made on the Sia blockchain. The user also pays the cost of the file contract in advance and then deducts a certain amount for the storage space in use. At the end of the file contract, the storage provider must prove that they save the user's data. This is called storage proof, and if it is satisfactorily provided within the period, a certain amount of Siacoin, paied by the user, is delivered to the storage provider. Merkle tree technology is used for proof of storage. 
Storj is a decentralized storage platform based on Ethereum blockchain technology that embraces architectural design elements from both centralized and decentralized networks. The data is partitioned by Reed-Solomon erasure coding technology after AES encryption, and each chunk is hashed and saved via a decentralized network[18]. Data integrity verification uses the Merkle tree created by data chunks. The verification is performed at regular intervals, and successful proof leads to a reward with cryptocurrency STORJ.

Unlike other decentralized storage platforms, Storj uses a centralized server for communication control. This so-called 'Bridge Server' allows certification of user information and quick selection of storage providers who will save an encrypted data chunk. Every element in the network interacts with the bridge server and all forms of communication are delegated by the bridge server, except for data transferred between users and storage providers. All storage providers and users gain access to the network through the bridge[19].

\subsection{NeoFS}

NeoFS is a distributed, decentralized storage platform developed by Neo SPCC. Built with Neo blockchain integration in mind, NeoFS aims to support the shift away from third-party storage providers, providing users with complete control over their data[20]. NeoFS uses the cryptocurrency Payment for storage services in NeoFS is only possible once a certain amount of Neo GAS has been deposited to the NeoFS smart contract address. The network searches for a storage provider who will save the data in consideration of the set cost.

NeoFS uses network maps and special data placement functions to save data. A network map is a multi-dimensional graph in which nodes have attributes and are grouped by those attributes and their values. Nodes in the network map have attributes such as location, storage type, and capacity. This finds nodes in the network to save data automatically without selecting a node.

Homomorphic cryptography is used for data encryption to order to increase confidence in data security. This is a technique used to operate on encrypted data without decrypting it. If the calculated result value is decrypted in the encrypted state, it obtains the same value as a result calculated with the existing data. This enables data integrity verification without decrypting the data[21]. 


\subsection{SAFE Network}

The SAFE network is a decentralized storage platform in which the Safecoin is used for payment method. Safecoin is not a cryptocurrency based on blockchain technology. One of the most commonly cited issues is the problem of scalability. While not a big problem in the initial phases of the network, it may hinder expansion since the size of the blockchain sharing is due to increase as the number of users of the network increases[22].

All data is self-encrypted before it reaches the SAFE network. The process is automatic and is carried out instantaneously. Self-encryption refers to the process by which a data chunk is split and then each chunk is encrypted using the other chunks of the same data chunk. The data map contains the locations of each chunk that makes up the data. The data map, with hashes before and after encryption, is used when retrieving and decoding the user data, as the encryption process is nonreversible[23].

Data integrity verification is performed by scoring nodes through the "proof of resource." The proof of resource measures the ability of a node to retrieve or store chunks of data based on its CPU speed, bandwidth availability, disk space, and online time. It is a way of ensuring that all network nodes are up to scratch. Failure to reach that standard leads to the automatic removal of the node from the network[24].

\section{Comparison of Various Decentralized Storage Platforms}

[Table 1] Comparison of Various Decentralized Storage Platforms

\begin{tabular}{|c|c|c|c|c|}
\hline & Blockchain & $\begin{array}{l}\text { Information } \\
\text { on chain }\end{array}$ & Data Verification & Encryption \\
\hline IPFS & $x$ & $x$ & $x$ & AES, Hashing \\
\hline Filecoin & Filecoin & $\begin{array}{l}\text { Assets, Transaction, } \\
\text { Contracts, Proofs }\end{array}$ & $\begin{array}{l}\text { PoSt (Proof-of-Spacetime), } \\
\text { PoRep (Proof-of-Replication) }\end{array}$ & AES, Hashing \\
\hline Sia & Ethereum & Assets, Transaction & $\begin{array}{c}\text { PoS (Proof-of-Storage), } \\
\text { Merkle tree }\end{array}$ & $\begin{array}{l}\text { Threefish, } \\
\text { Hashing }\end{array}$ \\
\hline Storj & Sia & $\begin{array}{l}\text { Assets, Transaction, } \\
\text { Contracts, Proofs }\end{array}$ & $\begin{array}{c}\text { File Contract } \\
\text { (Merkle root hash) }\end{array}$ & $\begin{array}{c}\text { AES, } \\
\text { Hashing }\end{array}$ \\
\hline NeoFS & $\mathrm{Neo}$ & $\begin{array}{l}\text { Assets, Transaction, } \\
\text { Contracts }\end{array}$ & $\begin{array}{c}\text { Homomorphic } \\
\text { Hashing }\end{array}$ & Homomorphic \\
\hline $\begin{array}{c}\text { SAFE } \\
\text { Network }\end{array}$ & $x$ & $x$ & PoS (Proof-of-Resource) & $\begin{array}{c}\text { Self-encryption, } \\
\text { Hashing }\end{array}$ \\
\hline
\end{tabular}

The table above summarizes the technologies required to develop decentralized storage 
platforms. The goals and use of the decentralized storage platforms under exam are mostly the same, but the methods for developing them differ. As a result, decentralized storage platforms are classified according to the presence of blockchain technology, and in case, according to the existence of their own blockchain. In addition, when transactions are made for storage, the contents of the transactions are saved in the blockchain, and there is a decentralized storage platform in which additional smart contracts or storage proof results are saved together. Various methods of proof are applied to verify the integrity of data saved in the storage, but most of the platforms use Merkle Tree technology. Data is usually encrypted and then decentralized via a network in the process of saving it in the storage. The encryption method was different for each decentralized storage platform.

\section{Conclusion}

In the present study, we derived the requirements and basic elements for decentralized storage platforms. We then compared the major decentralized storage platforms in terms of blockchain usage, data decentralization method, data encryption method, and data integrity verification. Various methods for proof of storage were applied to verify the integrity of data stored on a decentralized network across multiple locations. To enable only data owners to access their data, decentralized storage platforms encrypt data and the data owner holds the encryption key. As a result, we found that a decentralized storage platform requires an extra function of security and protection for data and has the potential to provide self-sovereign data. In the future, we plan to perform a quantitative comparison of the decentralized storage platforms discussed in this paper. In addition, we will also continue to investigate the issues encountered by decentralized storage platforms in providing an ecosystem of self-sovereign data.

\section{References}

[1] https://medium.com/@memolabs/centralized-storage-v-s-decentralized-storage-bc4f30504e75, Aug 25 (2019)

[2] Yunmook Nah, Cloud computing issues and current status, KEIT PD ISSUE REPORT, Dec (2011), Vol.10

[3] https://www.geeksforgeeks.org/comparison-centralized-decentralized-and-distributed-systems/?ref-rp, Dec 24 (2018)

[4] https://namu.wiki/w/Dropbox, Jan 28 (2020)

[5] https://www.devteam.space/blog/how-to-build-a-decentralized-cloud-storage-solution-like-storj-io/, Dec 24 (2019) 
[6] Zuojie Deng and Xiaolan Tan, A Remote File Possession Checking Protocol in a Cloud Storage, International Journal of Future Generation Communication and Networking, (2015), Vol.8, No.6, pp.115-122. DOI: $10.14257 /$ ijfgen.2015.8.6.11

[7] Ning Han, An Energy-Efficiency Enhanced Mobile Storage Platform in Cloud Environments, International Journal of Future Generation Communication and Networking, (2016), Vol.9, No.6, pp.87-98. DOI: 10.14257/ijfgcn.2016.9.6.09

[8] Cai Liang and Huang Hao, Design and Research on the Special System Architecture for Peer-to-Peer Distributed Storage System Based on Node Grouping, International Journal of Future Generation Communication and Networking, (2017), Vol.10, No.1, pp.271-280. DOI: 10.14257/ijfgen.2017.10.1.23

[9] Dylan Yaga, Peter Mell, Nik Roby, Karen Scarfone, Draft NISTIR 8202 Blockchain Technology Overview, Jan (2018) DOI: 10.6028/NIST.IR.8202

[10] https://sia.tech/technology, Apr 11 (2020)

[11] https://www.blockchainhub.kr/bbs/board.php?bo_table=lecture\&wr_id=1361, Apr 19 (2018)

[12] https://steemit.com/kr/@bigthumbsup/genaro-network, Jun 04 (2018)

[13] https://docs.google.com/document/d/1juk_ByLdaggZuz6k5y6n90R7FqK2Gn1qrh38sbNapHw/edit, Feb 19 (2019), Beyond code, Legal Block White Paper Version 4.0

[14] Juan Benet, IPFS - Content Addressed, Versioned, P2P File System (DRAFT 3), Jul 24 (2014) DOI: 10.48550/arXiv.1407.3561

[15] https://www.slideshare.net/chenghanTsai1/ipfs-85264010, Dec 29 (2017)

[16] https://brunch.co.kr/@minqukim/12, Apr 04 (2018)

[17] David Vorick, Luke Champine, Sia: Simple Decentralized Storage, blockchainlab, Nov 29 (2014)

[18] https://github.com/storj/whitepaper, Oct 30 (2018), STORJ, Storj: A Decentralized Cloud Storage Network Framework

[19] Xiaolu Zhang, Justin Grannis, Ibrahim Baggili, Nicole Lang Beebe, Frameup: An incriminatory attack on Storj: A peer to peer blockchain enabled distributed storage system, Digital Investigation, (2019), Vol.29, pp.28-42. DOI:10.1016/j.diin.2019.02.003

[20] https://fs.neo.org/, Mar 04 (2020)

[21] http://wiki.hash.kr/index.php/HE, Aug 05 (2019)

[22] https://medium.com/safenetwork/consensus-without-a-blockchain-1dfda94aa435, Jan 29 (2015)

[23] https://safenetwork.tech/how-it-works/, Mar 04 (2020)

[24] https://safenetforum.org/t/proof-of-resource-on-the-safe-network/24404, Jul 05 (2018) 